\title{
Self-assembled microtubes and rhodamine 6G functionalized Raman-active gold microrods from 1-hydroxybenzotriazole
}

\author{
RAVULA THIRUPATHI ${ }^{\mathrm{a}, \mathrm{b}}$ and ERODE N PRABHAKARAN ${ }^{\mathrm{b}, \mathrm{c}, *}$

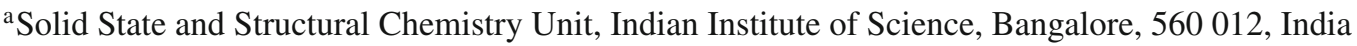 \\ ${ }^{\mathrm{b}}$ Nanoscience Initiative, Indian Institute of Science, Bangalore, 560 012, India \\ ${ }^{\mathrm{c}}$ Department of Organic Chemistry, Indian Institute of Science, Bangalore, 560 012, India \\ e-mail: eprabhak@orgchem.iisc.ernet.in; erodeprabhakaran@gmail.com
}

MS received 18 October 2010; revised 20 January 2011; accepted 4 February 2011

\begin{abstract}
Hydroxybenzotriazole spontaneously self-assembles to form hollow, linear microtubes initiated by controlled evaporation from water. The tube cavities act as thermo-labile micromoulds for the synthesis of linear gold microrods. Rhodamine 6G-labelled gold microrods, exhibiting surface enhanced resonance Raman activity, have been synthesized using the HOBT microtubes.
\end{abstract}

Keywords. Microtubes; gold microrods; self-assembly; Raman spectroscopy; 1-hydroxy benzotriazole.

The design and identification of small organic molecules that spontaneously self-assemble into hollow tubular structures ${ }^{1,2}$ has received great attention over the past decade. Several hetero-oligomeric building blocks such as amphiphiles, ${ }^{3,4}$ conjugated aryl macrocyles, ${ }^{5}$ peptides ${ }^{6,7}$ and carbohydrate derivatives ${ }^{8,9}$ and aryl amides, ${ }^{10}$ have been identified to self-assemble into hollow tubular structures under suitable conditions. Controlling the dimensions and characteristics of these tubes and their inner cavities however is not easy and is usually achieved in two ways. One is where functional groups like phenyl rings, amide bonds, aliphatic chains, thiols, and charged groups, which have high propensity to self interact, are liberally introduced on the surfaces of large self-assembling monomeric building blocks. ${ }^{4}$ The other way is to take advantage of the sheet-like crystal packing in certain molecules and direct their folding into hollow tubular structures, in appropriate solvents. ${ }^{11}$ The latter method has been most effective in identifying the smallest self-assembling organic molecules like the diphenylalanine dipeptide ${ }^{11-13}$ and its derivatives. ${ }^{6,14-16}$ While these small peptide-based tubes have potential applications as templates for casting metals, they have the disadvantage of being easily recognized and degraded by physiological enzymes. ${ }^{7}$ Small non-peptide organic molecules that can similarly self-assemble into hollow tubular structures and

\footnotetext{
${ }^{*}$ For correspondence
}

encapsulate metals, on the other hand, will have more bioavailability and hence have broader applications in medicine, ${ }^{17}$ biosensing ${ }^{18}$ and molecular electronics, ${ }^{19}$ than their peptide counterparts. However, there are no reports of the self-assembly of such small non-peptide organic molecules.

In this endeavour, we screened several small nonpeptide molecules for their propensity to spontaneously self-assemble into tubular structures. We have identified 1-hydroxybenzotriazole monohydrate (HOBT) as the smallest non-peptide organic molecule to self-assemble into hollow tubular structures on controlled evaporation from aqueous solutions. The tube formation occurs by the rolling over of crystalline sheets of HOBT. The molecular packing of HOBT in these tubes has been characterized. The tube cavities have been used as molds to synthesize gold microrods with homogenous dimensions and dye-functionalized surfaces.

HOBT is sparingly soluble in water and crystallizes as continuous sheets from water ${ }^{20}$ at concentrations above $2 \mathrm{mg} \mathrm{mL}^{-1}$. Evaporation of water from aqueous solutions of $\mathrm{HOBT}\left(1-2 \mathrm{mg} \mathrm{mL}^{-1}\right)$ at $20 \pm 2^{\circ} \mathrm{C}$ on silicon wafers yielded highly ordered and patterned linear horizontal microtubes with polydisperse thickness $(1-8 \mu \mathrm{m})$ and persistence lengths of several hundreds of $\mu \mathrm{m}$ (figure 1a). Magnified SEM images of the tubes revealed that they were open-ended (figure 1f) and their wall thickness varied from 0.4 to $2 \mu \mathrm{m}$ (figure 1c). Interestingly, several partially and incompletely rolled-over cylindrical structures were also 

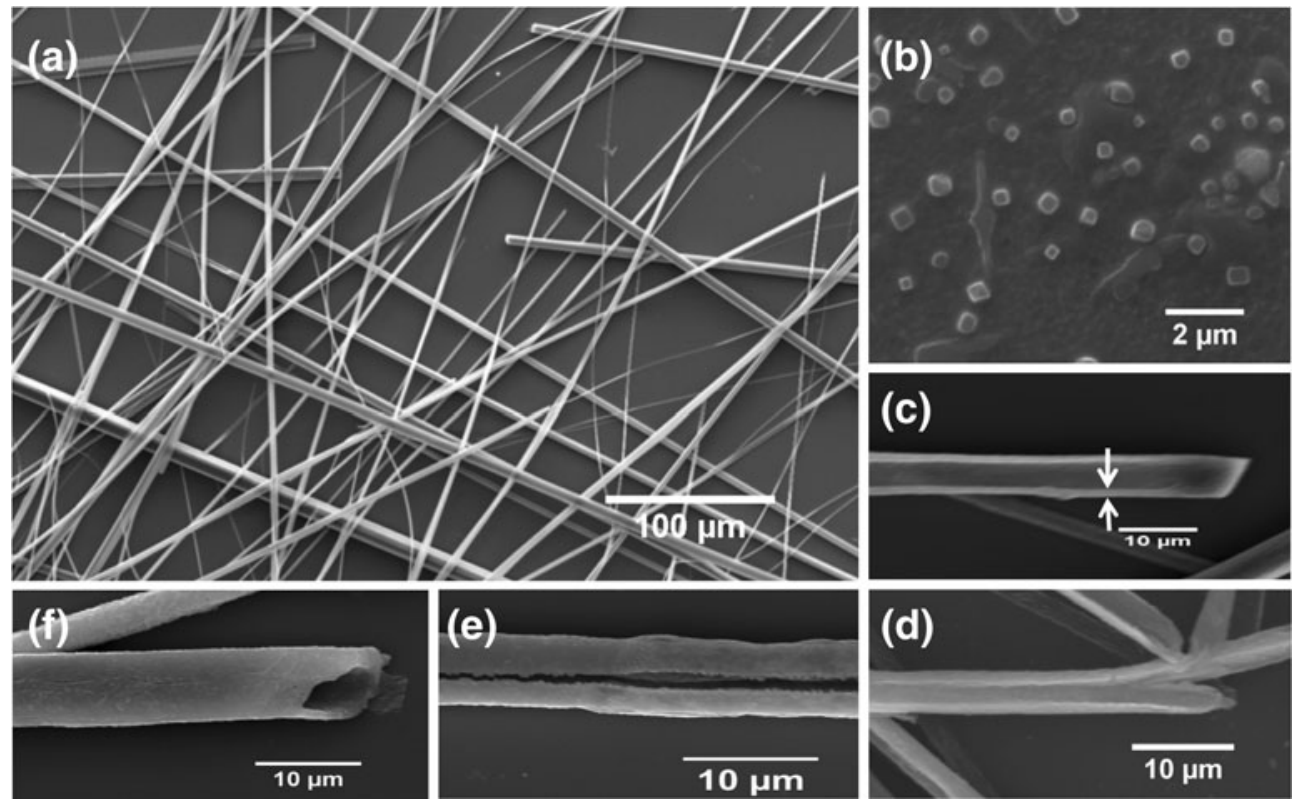

Figure 1. SEM images of the hollow microtubes of HOBT. (a) HOBT microtubes formed by evaporation of water at $20 \pm 2^{\circ} \mathrm{C}$ from $1 \mathrm{mg} \mathrm{mL}^{-1}$ aqueous solution of HOBT. (b) Microcube structures formed from $10 \mu \mathrm{g} \mathrm{mL} \mathrm{mL}^{-1}$ aqueous solution of HOBT; (c-e) Incompletely rolled-over sheets of HOBT. (f) Magnified SEM image of hollow end of a HOBT microtube.

formed (figures 1c-e) along with the fully formed microtubes. In several of these partially rolled structures, the rollover of sheets is clearly seen to occur along the length of the incipient cylindrical tube (figure 1e). Formation of these structures suggests that the microtubes are formed by the rolling over of sheets of HOBT (0.4 to $2 \mu \mathrm{m}$ thickness). Similar tube formation is also observed from aqueous methanolic solutions of HOBT (figure S1). Interestingly, only plain sheets of HOBT are formed from anhydrous methanolic solutions of HOBT (figure S2). Hence water is crucial for the rollover process. Moreover, evaporation of aqueous solutions of HOBT with very low initial concentrations $\left(10 \mu \mathrm{m} \mathrm{mL}^{-1}\right)$ at $20^{\circ} \mathrm{C}$, exclusively yielded cube-shaped structures (figure $1 \mathrm{~b}$ ), whose dimensions (250-400 nm) were smaller than those of the laminar walls of the microtubes. It is possible that initial formation of HOBT sheets results from self-attachment of these cubic structures at higher initial concentrations. While the mechanistic role of water in the rollover process is not yet clear, it is possible that the polarity difference between the bulk water and the silicon wafer surface contributes to the rollover process. Although such rollover mechanism has been proposed for the formation of peptide nanotubes, ${ }^{11}$ the current images provide the first visual proof of that mechanism operating in the formation of self-assembled organic microtubes.
The powder X-ray diffraction spectra of the HOBT microtubes matched exactly with that of the crystals of HOBT obtained from water (figure 2a) implying similar molecular arrangements ${ }^{20}$ in both. The HOBT molecules are stacked in columns running along the crystallographic $c$-axis and stabilized by $\pi$-stacking interactions with typical centroid-centroid distances of $<3.5 \AA$ between the benzene rings. ${ }^{20}$ Water molecules reside in the small pockets between the columns, each one involved in three hydrogen bonding interactions (HBIs) - two as donors and one as acceptor - with three HOBT molecules. In all the three molecules the oxide anion $\left(-\mathrm{O}_{10^{-}}\right)$of HOBT acts as the hydrogen bond acceptor from water and the $\mathrm{N}_{3}-\mathrm{H}$ acts as the donor (figure 3). Although the microtubes homogenously contain the hydrated ionic (1-oxide) tautomer (figure 2d) of HOBT, the hydroxy group is crucial for its ordered self-assembly into microtubes. This is seen from the fact that benzotriazole, which lacks the hydroxy group of HOBT but can also hydrogen bond with water through its $\mathrm{NH}$ group, forms nondisceretely oriented low aspect ratio microtubes under similar experimental conditions, while $\mathrm{NaOBT}^{21}$ (the sodium salt of HOBT) forms only irregular sheet-like structures (figure 4).

In spite of the identical packing of HOBT around the water molecules in the microtubes and in the crystals, 

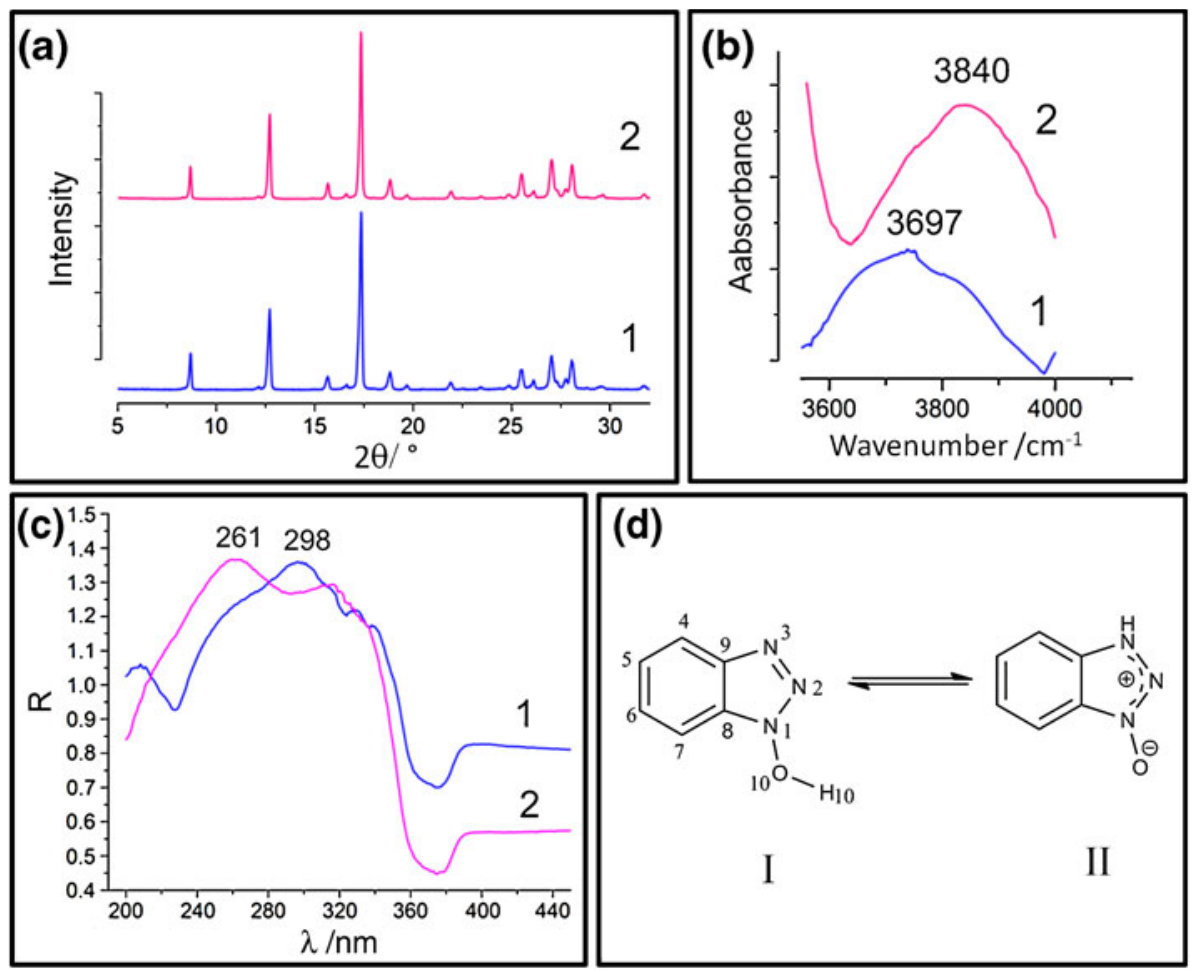

Figure 2. Spectral characterization of (1) HOBT crystals from water and (2) HOBT microtubes: (a) Powder X-ray diffraction spectra of 1 and 2. (b) The O-H absorption in the FT-IR spectra of 1 and 2. (c) UV-vis reflectance spectra of thin films of 1 and 2. (d) The two prototropic tautomeric structures of HOBT with atom numbering: (I) the $N$-hydroxy tautomer and (II) the benzotriazole-1-oxide tautomer.

the hydroxyl group of HOBT interacts differently with water in the former and the latter. Comparison of the FT-IR spectra ${ }^{22}$ of the crystals with that of the microtubes revealed differences exclusively in the absorption bands for the packed water molecules (figure S3). The water absorption band at $3697 \mathrm{~cm}^{-1}$ in the crystals is blue shifted by $143 \mathrm{~cm}^{-1}$ to $3840 \mathrm{~cm}^{-1}$ in the microtubes (figure $2 \mathrm{~b}$ ). Thus the packed water molecules in the microtubes are less involved in hydrogen bonding interactions with the HOBT molecules than in the (a)

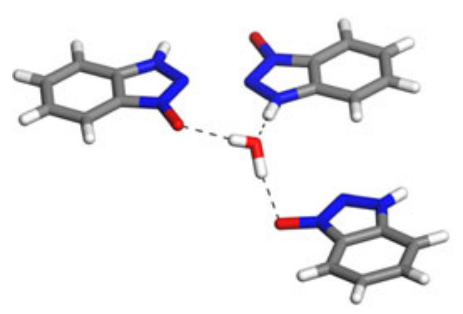

(b)

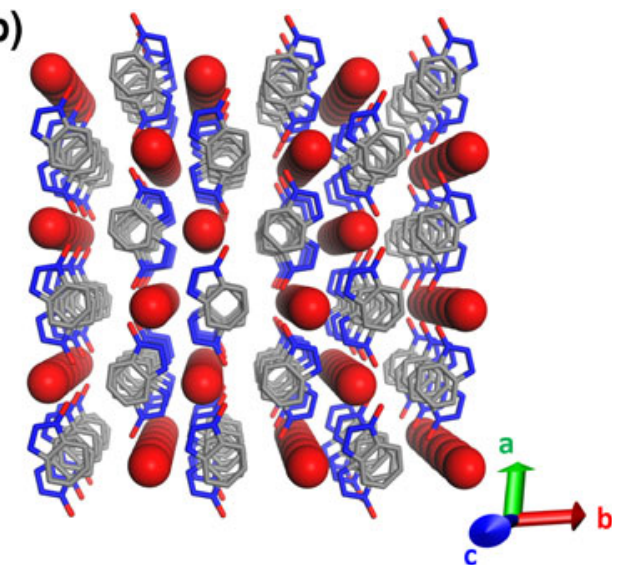

Figure 3. (a) Structure of one water molecule hydrogen bonded (2 as donors and 1 as acceptor) with three ionic 1-oxide tautomers (II) of HOBT in the microtubes. (b) Packing of the aromatic rings through $\pi$-stacking interactions along the crystallographic $c$-axis. The oxygen of $\mathrm{H}_{2} \mathrm{O}$ is shown as a red sphere. Hydrogens have been removed for clarity. 

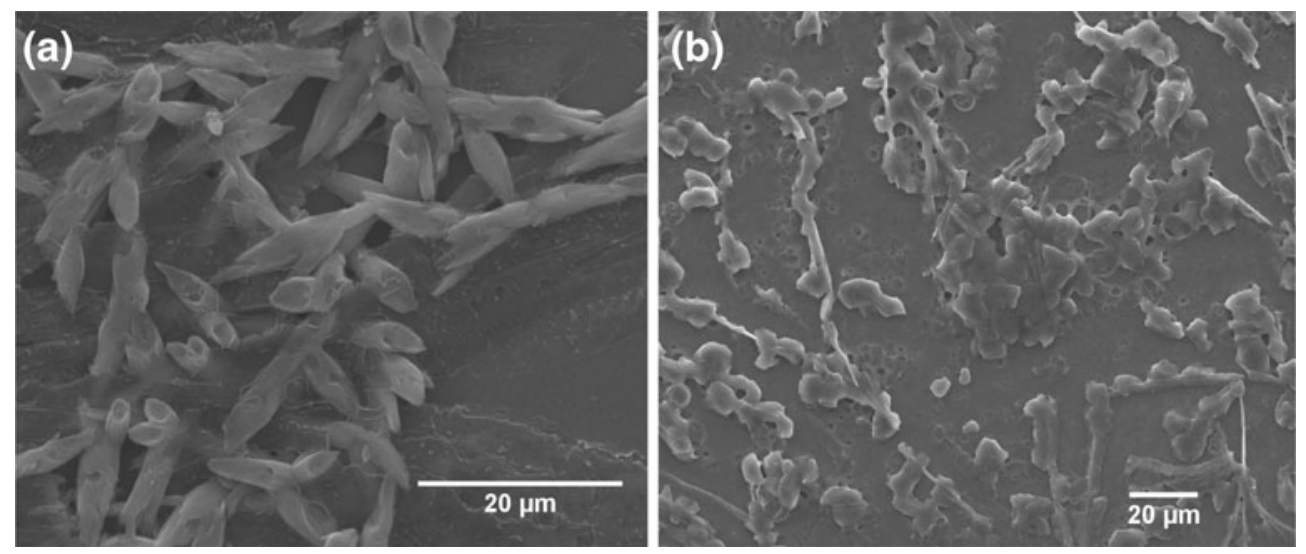

Figure 4. (a) SEM image of benzotriazole formed by evaporation-initiated growth of HOBT from an aqueous solution $\left(1 \mathrm{mg} \mathrm{mL}^{-1}\right)$. (b) SEM image of NaOBT formed by evaporation-initiated growth from water $\left(1 \mathrm{mg} \mathrm{mL}^{-1}\right)$.

crystals. The $\lambda_{\max }$ in the UV-vis reflectance spectra for the dry thin films of the HOBT microtubes (figure 2c) is also significantly blue shifted to $261 \mathrm{~nm}\left(\pi \rightarrow \pi^{*}\right.$ transition) from $298 \mathrm{~nm}$ for the crystals. Such a shift is indicative of a significantly less polar environment, similar to that of ethanol ${ }^{23}$ around HOBT in the microtubes. This further indicates the relatively poor association of the packed water molecules with the HOBT molecules in the microtubes and perhaps also explains their significantly lower melting point $\left(140^{\circ} \mathrm{C}\right)$ compared to the crystals $\left(157^{\circ} \mathrm{C}\right)$. Together, these results suggest that the HOBT microtube structure is predominantly held together by crystalline intermolecular $\pi$-stacking interactions between the aryl groups very similar to those found in much larger self-assembling monomers like resorcenarenes ${ }^{8}$ and aryl macrocycles. ${ }^{5}$
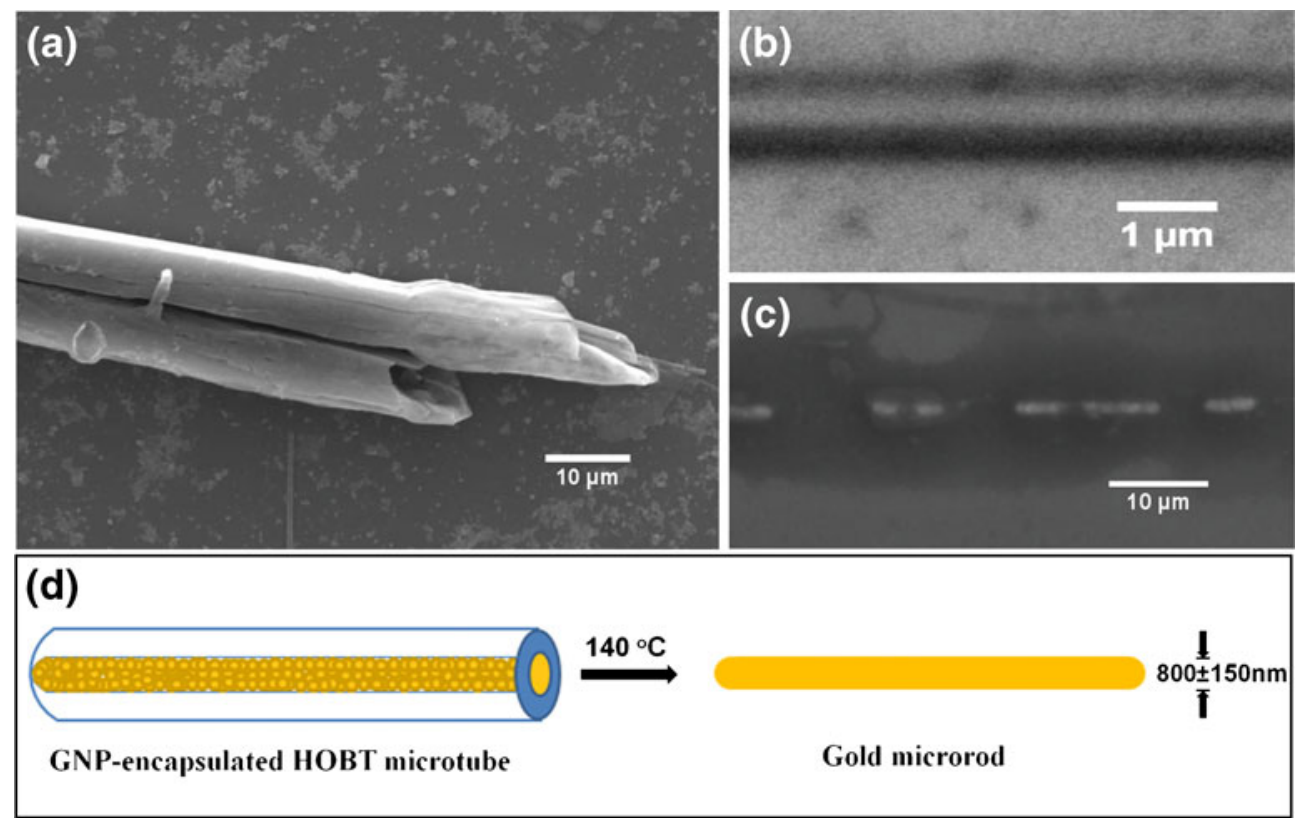

Figure 5. Synthesis of gold microrods: (a) Magnified SEM image of a pair of closely selfassembled, well-separated open-ended microtubular structures synthesized by evaporation of a sonicated aqueous mixture $(1: 1 \mathrm{v} / \mathrm{v})$ of HOBT $\left(1 \mathrm{mg} \mathrm{mL}^{-1}\right)$ and GNPs $\left(10^{10}\right.$ particles $\left.\mathrm{mL}^{-1}\right)$ (8-10 nm diameter). (b) Magnified SEM image of the surface of a gold microrod. (c) Discontinuous sausage-like linear arrays of gold microrods with uniform diameters. (d) Schematic of the synthesis of gold microrods (yellow) by melting of the gold encapsulated HOBT microtube moulds at $140^{\circ} \mathrm{C}$. 
This is remarkable in such a small molecule and HOBT could represent the smallest molecule with optimum balance between the surface area and the energy of the intermolecular interactions for efficient tubular self-assembly.

One of the significant advantages of hollow tubular structures derived from self-assembly of small molecules is their potential to encapsulate metal nanoparticles in their inner cavities with greater facility than the carbon nanotubes. Incubating a solution of HOBT (1-2 mg mL ${ }^{-1}$ ) with a suspension of gold nanoparticles $^{24}$ (GNPs) (8-10 nm diameter; $10^{8}-10^{11}$ particles $\mathrm{mL}^{-1}$ ) followed by evaporation of water at 20 $\pm 2^{\circ} \mathrm{C}$ resulted in formation of microtubes (figure 5a) with polydisperse diameters $(0.5-12 \mu \mathrm{m})$ (figure S4). Energy-dispersive X-ray spectroscopy (EDX) analysis revealed absence of gold on the tube surface. But melting the HOBT microtubes by incubating them at $140^{\circ} \mathrm{C}$ (10 minutes) resulted in long, linear arrays of gold rods (see figure S5 for EDX) with monodisperse thickness
$(800 \pm 150 \mathrm{~nm})$ and smooth surface (figure 5b). A few short and discontinuous linear arrays of gold rods $(800 \pm 150 \mathrm{~nm}$ width) of irregular length were also formed (figure 5c). These structures suggest that the microtube cavities act as non-interacting molds for encapsulation of the GNPs (figure 5d). Further, formation of the discontinuous rods indicates that GNP encapsulation occurs during the rollover of the HOBT sheets, rather than after. The selective encapsulation of the neutral GNPs within the cavities of HOBT tubes is very unlike the peptide-based nanotubes where the GNPs adhere to the outer convex surface of the tubes. ${ }^{11}$ Further, since the HOBT micromolds melt at a relatively low temperature $\left(140^{\circ} \mathrm{C}\right)$ compared to peptide based tubes ${ }^{13}\left(>250^{\circ} \mathrm{C}\right)$, they can have applications for the synthesis of metal-organic hybrid rods.

The strong insertion of the triazole motif in benzotriazoles into the surface of copper and gold has been very well established. ${ }^{25}$ Such insertion is indicated by the enhancement of the Raman spectral signals for
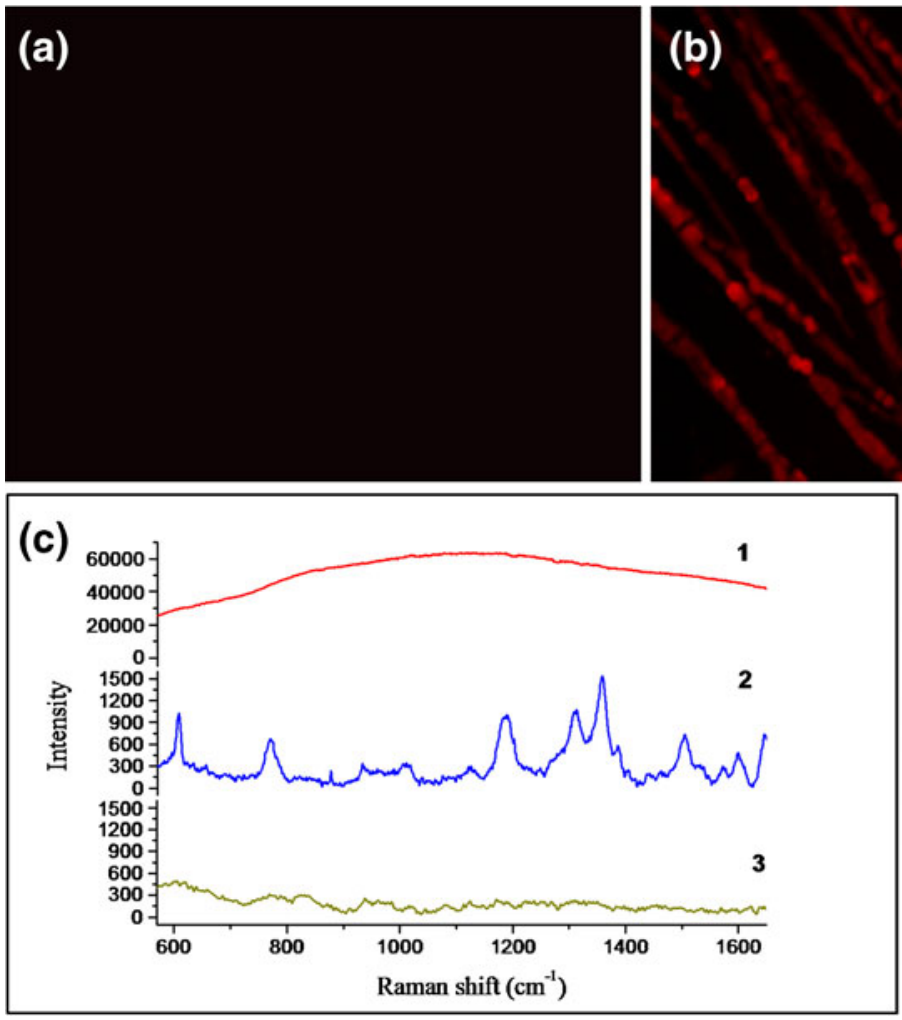

Figure 6. Fluorescence microscope image of (a) gold microrods synthesized using HOBT microtubes as molds. (b) Rhodamine 6G (R6G)-functionalized gold microrods. (c) Surface enhanced Raman spectrum of thin film of (1) aqueous solution of R6G $(0.1 \mathrm{mM})$; (2) the gold microrods with R6G functionalized on the microrod surface and (3) suspension of only the gold microrods. (d) Image under visible light of aqueous suspensions of (1) the gold microrods and (2) the gold microrods with R6G functionalized on the microrod surface in eppendorf vials.
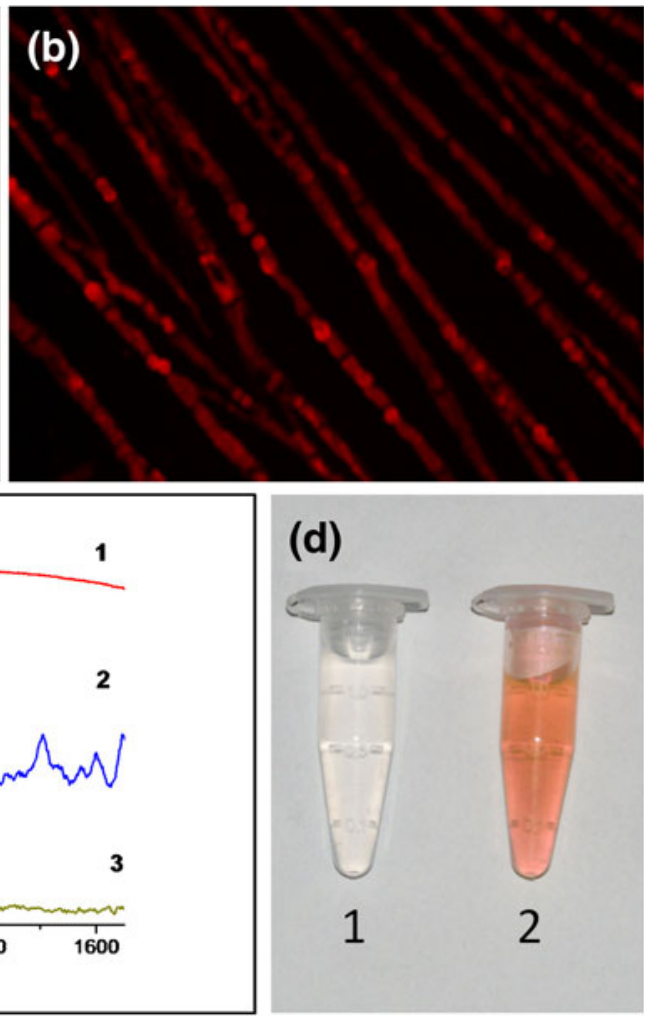
triazoles when mixed with these metal nanoparticles. Most studies of HOBT on the other hand, have explored only its ability to form excellent coordination complexes with various metals. ${ }^{26}$ Insertion of the $N$ hydroxytriazole ring on metal surfaces has not been reported. We found that the Raman spectral signals and intensities (figure S6) for a mixture of HOBT (1 mg $\left.\mathrm{mL}^{-1}\right)$ and the GNPs $\left(10^{12}\right.$ particles $\left.\mathrm{mL}^{-1}\right)$ in water are exactly similar to that of a solution of HOBT in water $\left(1 \mathrm{mg} \mathrm{mL}^{-1}\right)$, clearly suggesting the lack of any interaction between HOBT and the GNPs. Hence the crystalline tube walls of HOBT seem to provide a non-interacting surface for encapsulation of the GNPs.

We envisioned to use the non-interacting surface of the HOBT microtubes to encapsulate surfacefunctionalized GNPs to synthesize tailor-made fluorescent gold microrods which can find applications as templates in ELISA and as single molecule biosensors in genotyping. ${ }^{27}$ Rhodamine 6G (R6G) is an organic cationic dye that strongly interacts with the electronrich surface of $\mathrm{GNPs}^{28}$ and is extensively used in ELISA. Addition of GNPs $\left(10^{12}\right.$ particles $\left.\mathrm{mL}^{-1}\right)$ to a solution of R6G $(0.1 \mathrm{mM})$ and HOBT $\left(2 \mathrm{mg} \mathrm{mL}^{-1}\right)$ in water resulted in the complete quenching of the high intensity (60000 a.u) fluorescence of R6G (figure $6 c(1))$. Evaporation of water from this aqueous mixture on silicon wafer at $20^{\circ} \mathrm{C}$, followed by heating of the resulting microtubes at $140^{\circ} \mathrm{C}$ for 10 minutes resulted in the formation of gold microrods that were functionalized with R6G on their surface as seen in their fluorescence microscope images (figure $6 \mathrm{~b}$ ) obtained using green excitation filter (480-550 nm). These microrods also exhibited Raman spectral signals of R6G (figure $6 \mathrm{~d}(2)$ ) indicating that R6G was bound on the GNP surface. As a control it was noted that the gold microrods themselves did not exhibit any Raman signals (figure $6 \mathrm{~d}(2)$ ) or fluorescence (figures 6a and c(3)).

Based on the above observations, a schematic of the mechanism for the formation of HOBT microtube structures and the encapsulation of the GNPs inside their cavities can be conceived (figure 7). Accordingly, crystalline microcubic structures of HOBT are formed during initial stages of evaporation of water. In the 1-oxide form all atoms in HOBT are in a single plane, (a)

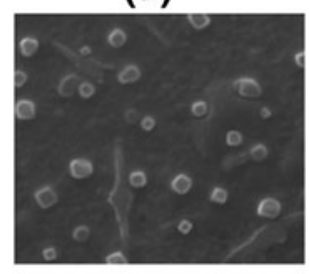

(d)

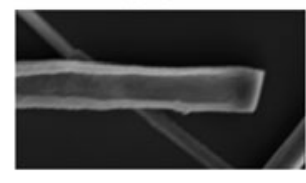

(g)

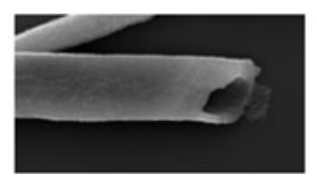

(b)

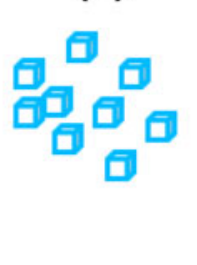

(e)

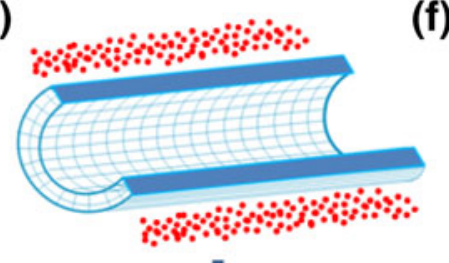

(h)

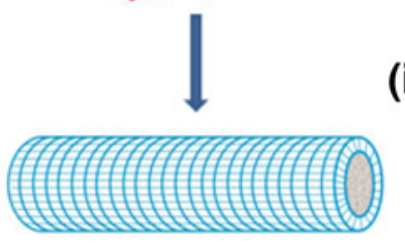

(i) (c)

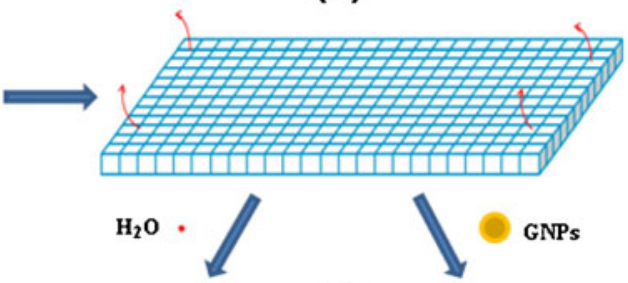

(f)
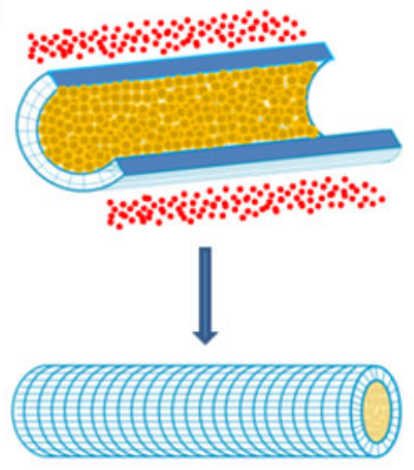

Figure 7. Schematic representation of the proposed mechanism for rolling over of HOBT sheets. (a) Formation of microcube structures from $0.1 \mathrm{mg} \mathrm{mL}^{-1}$ aqueous solution of HOBT. (b) Schematic representation of cubic structures of HOBT and (c) the crystalline sheets formed by self-attachment of the cubes. (d) HR-SEM image of a half-rolled microsheet structure. (e) Schematic representation of a half-rolled sheet in water and (f) the agglomeration of GNPs inside the concave cavity of a half-rolled sheet. (g) HR-SEM of the open end of a HOBT microtube, evaporated from $1 \mathrm{mg} \mathrm{mL}^{-1}$ aqueous solution. (h) Schematic representation of a rolled hollow microtube and (i) a GNP encapsulated microtube. 
which removes the possibility that the crystal packing is initiated by any of the three conformations that are possible for the hydroxy form of the HOBT molecule. ${ }^{29}$ The cubic structures then self-attach to form sheet structures at higher concentrations. The crystalline sheets rollover in water to form the microtubular structures. The exact role played by water in the formation of such microstructures is not clear. However, it is interesting to note that the rollover process does not occur in the less polar solvent methanol. When GNPs (or surface-functionalized GNPs) are present in the aqueous medium, they encapsulate preferentially within the concave wall surface of the forming HOBT half tubes, probably owing to the difference in polarity between the bulk solvent and the incipient tube interior. Heating of the resulting tubes leads to agglomeration of the GNPs and melting of the outer HOBT micromold and the eventual formation of the gold microrods.

The present work has identified HOBT as the smallest non-peptide building block that spontaneously selfassembles into hollow tubular structures upon evaporation from water. The tubes form under ambient conditions by rolling over of crystalline sheets of HOBT. The packing of HOBT in the tubes seem to be predominantly driven by intermolecular $\pi$-stacking interactions between the aromatic rings of HOBT. These structural and packing patterns are similar to those found in nanotubes formed by the self-assembly of peptides and other larger molecules. The cavities of these thermolabile microtubes act as molds for casting gold nanoparticles for the synthesis of gold microrods with monodisperse dimensions. The non-reacting inner surfaces of the cavities have been used to uniquely synthesize R6G-functionalized gold microrods. With these features, HOBT is an important, novel, non-peptide building block for accessing micro and nanometric materials for their applications in medicine, biology and molecular biotechnology.

\section{Supplementary information}

Materials and methods, figures S1, S2, S3, S4, S5 and S6 are given as supplementary information (see www.ias.ac.in/chemsci).

\section{Acknowledgements}

We are grateful to Prof. N Chandrabhas and Mr. S Soumik of Jawaharlal Nehru Centre for Advanced Scientific Research (JNCASR), Bangalore, for providing us with the GNPs, R6G and for helping with the SERRS experiments. We thank the Institute Nanoscience Initiative and Prof. T N Gururow, Indian Institute of Science (IISc), for providing us access to the electron microscopes and X-ray diffractometer respectively. We are grateful to Prof. S Natarajan for the valuable and informative discussions. We thank the Council of Scientific and Industrial Research (CSIR), India, for providing partial funds for this project. RT thanks CSIR for his Junior Research Fellowship.

\section{References}

1. Gazit E 2007 Chem. Soc. Rev. 361263

2. Rajagopal K and Schneider J P 2004 Curr. Opin. Struct. Biol. 14480

3. Vauthey S, Santoso S, Gong H Y, Watson N and Zhang S 2002 Proc. Natl Acad. Sci. USA 995355

4. Hartgerink J D, Beniash E and Stupp S I 2001 Science 2941684

5. Shetty A S, Zhang J and Moore J S 1996 J. Am. Chem. Soc. 1181019

6. Gupta M, Bagaria A, Mishra A, Mathur P, Basu A, Ramakumar S and Chauhan V S 2007 Adv. Mater. (Weinheim, Ger.) 19858

7. Reches M and Gazit E 2003 Science $\mathbf{3 0 0} 625$

8. Sun Y, Yan C-G, Yao Y, Han Y and Shen M 2008 Adv. Funct. Mater. 183981

9. Bonini M, Rossi S, Karlsson G, Almgren M, Lo Nostro $\mathrm{P}$ and Baglioni P 2006 Langmuir 221478

10. Bushey M L, Nguyen T-Q and Nuckolls C 2003 J. Am. Chem. Soc. 1258264

11. Reches M and Gazit E 2006 Nat. Nanotechnol. 1195

12. Reches M and Gazit E 2003 Science $\mathbf{3 0 0} 625$

13. Adler-Abramovich L, Reches M, Sedman V L, Allen S, Tendler S J and Gazit E 2006 Langmuir 221313

14. Panda J J, Mishra A, Basu A and Chauhan V S 2008 Biomacromolecules 92244

15. Akazome M, Ueno Y, Ooiso H and Ogura K 2000 J. Org. Chem. 6568

16. de Groot N S, Parella T, Aviles F X, Vendrell J and Ventura S 2007 Biophys. J. 921732

17. Abidian M, Kim D H and Martin D 2006 Adv. Mater. (Weinheim, Ger.) 18405

18. de la Rica R, Mendoza E, Lechuga L and Matsui H 2008 Angew. Chem., Int. Ed. 479752

19. de la Rica R and Matsui H 2010 Chem. Soc. Rev. 393499

20. Bosch R, Jung G and Winter W 1983 Acta Crystallogr., Sect. E: Struct. Rep. Online 391089

21. NaOBT was prepared by the reaction of HOBT hydrate with a molar equivalents of sodium methoxide in anhydrous methanol

22. Fagel J E and Ewing G W 1951 J. Am. Chem. Soc. 73 4360

23. Boyle F T and Jones R A Y 1973 J. Chem. Soc., Perkin Trans. 2160

24. In a typical procedure, a solution of $1 \%$ sodium citrate was added to a boiling aqueous solution of $\mathrm{HAuCl}_{4}$ and continued to boil for $1 \mathrm{~h}$, to yield a suspension of the 
GNPs. Lee P C and Meisel D 1982 J. Phys. Chem. 86 27. Graham D, Faulds K and Smith W E 2006 Chem. 3391

Commun. (Cambridge, U.K.) 4363

25. Graham D, McAnally G, Jones J C and Ewen Smith W 28. Michaels A M, Nirmal M and Brus L E 1999 J. Am. 1998 Chem. Commun. 1187 Chem. Soc. 1219932

26. Hammud H H, Holman K T, Masoud M S, El-Faham A 29. Kumar V P, Ganguly B and Bhattacharya S 2004 J. Org. and Beidas H 2009 Inorg. Chim. Acta 3623526 Chem. 698634 